\title{
High Deleterious Genomic Mutation Rate in Stationary Phase of Escherichia coli
}

\author{
Laurence Loewe, ${ }^{*} \dagger$ Volker Textor, Siegfried Scherer
}

\begin{abstract}
In natural habitats, bacteria spend most of their time in some form of growth arrest. Little is known about deleterious mutations in such stages, and consequently there is limited understanding of what evolutionary events occur. In a deleterious mutation accumulation experiment in prolonged stationary phase of Escherichia coli, about 0.03 slightly deleterious mutations were observed per genome per day. This is over an order of magnitude higher than extrapolations from fast-growing cells, but in line with inferences from observations in adaptive stationary phase mutation experiments. These findings may affect understanding of bacterial evolution and the emergence of bacterial pathogenicity.
\end{abstract}

Mutation rates and selection coefficients are fundamental for understanding evolutionary processes. Terumi Mukai was the first to determine both parameters for deleterious mutations in a population of fruit flies (1). Similar studies have led to considerable insights into spontaneous deleterious mutations (2-4). While most experiments targeted the fruit fly Drosophila melanogaster or the worm Caenorhabditis elegans, few have studied microorganisms. Aside from more general work on RNA viruses (5), deleterious mutation parameters have been investigated in yeast $(6,7)$, in Salmonella typhimurium (8), and in Escherichia coli (9-11). All of these studies have estimated mutation rates per generation during logarithmic growth but have not investigated stationary phase. A recent study on stress-induced mutations in aging colonies of $787 \mathrm{E}$. coli strains showed that the average frequency of mutations to resistance against the antibiotic rifampicin was sevenfold higher in 7-day-old colonies as compared to 1-day-old colonies (12), but the rate of deleterious mutations has not been measured.

In natural habitats, bacterial populations spend most of their time under nutritional constraints $(13,14)$, and rates of growth may be very slow, because essential nutrients are consumed much faster than they become available. At least in one $E$. coli assay system (the Lac frameshift reversion assay), to evade death by starvation under such conditions, a subpopulation can turn

Microbial Ecology Group, Department of Biosciences, Technische Universität München, Weihenstephaner Berg 3, 85354 Freising, Germany.

*To whom correspondence should be addressed. Email: Laurence.Loewe@evolutionary-research.net

$\uparrow$ Present address: Institute of Cell, Animal and Population Biology, University of Edinburgh, Ashworth Laboratories, Kings Buildings, West Mains Road, Edinburgh EH9 3JT, Scotland, UK. into transient mutators to increase the probability of an adaptive mutation arising that allows growth to resume on an alternative nutrient (15-17). In another assay system, cells with a growth advantage in stationary phase (GASP) repeatedly take over the population $(14,18)$. The mutational processes used in these systems differ mechanistically from those occurring during the logarithmic growth phase $(14,19)$, as recombination and DNA polymerases induced by SOS (a DNA damage response) appear to play a key role $(15,19,20)$. Cells in stationary phase might divide one to two times per day $(14,21)$ or even less than once per 3 days (22); however, to accumulate mutations they need neither to divide (23) nor to replicate their DNA globally (24). Given the importance of stationary phase in the naturally occurring life cycle of bacteria, the deleterious mutation rate during that phase could be crucial for understanding bacterial evolution. Here, we report on a Bateman-Mukai (BM) analysis of a stationary phase mutation accumulation experiment that estimates this rate (25).

Because it is difficult to estimate the number of generations per day in the stationary phase, we have abandoned the generation-centric view of mutation rates in favor of a clock-like view and have measured mutation rates per genome per day. Figure S1 depicts the design of a 101-day stationary phase mutation accumulation experiment with 98 replicate lines. Fitness was measured in terms of maximal growth rate $m$, and the resulting distributions of growth rate showed a decrease in mean and an increase in variance over time (Fig. 1), leading to a typical BM plot (Fig. 2). Depending on the distributions selected for estimating mutational parameters as well as the method used [BM or maximum-likelihood (ML)], a number of different values were obtained [supporting online material (SOM) Text]. The most reliable values are deleterious mutation rates of $\mathrm{U}_{\mathrm{BM}}=0.027$ and $\mathrm{U}_{\mathrm{ML}}=0.045 \pm 0.004$ per genome per day with deleterious selection coefficients of $\mathrm{s}_{\mathrm{BM}}=$ $3.4 \%$ and $\mathrm{s}_{\mathrm{ML}}=2.3 \pm 0.2 \%$, assuming that all mutations have the same effect [ML values with $95 \%$ confidence intervals (26)]. If mutational effects are distributed exponentially, then $\mathrm{U}_{\mathrm{ML}}=0.091 \pm 0.01$ per genome per day and $\mathrm{s}_{\mathrm{ML}}=1.1 \pm 0.1 \%$. Different shape parameters of a Gamma distribution gave equal or increasing likelihoods for more leptocurtic distributions leading to corresponding higher $U$ and lower s estimates. Therefore, inferences on the distribution of mutational effects are not possible here (26). It was important to allow longterm stationary phase lines to acclimate to logarithmic growth for at least one or two additional serial transfers, because temporal changes of gene expression can lead to much slower growth rates. In summary, the values to remember are $\mathrm{U} \approx 0.03$ mutations per genome per day with a selection coefficient of $\mathrm{s} \approx 3 \%$.

The selection coefficients found here are in general agreement with $\mathrm{s}_{\max } \approx 1.2 \%$ from the E. coli log-phase mutation accumulation experiment of Kibota and Lynch (9) and similar to the $2.7 \%$ reported for artificial random transposon insertions by Elena and Lenski (27). To understand the mutation rate found here is more challenging. Any purely replication-based model fails: Assuming two generations per day in stationary phase $(21)$ and $U_{\text {deleterious }}$ of 0.0002 mutations per genome per generation (9) results in one to two orders of

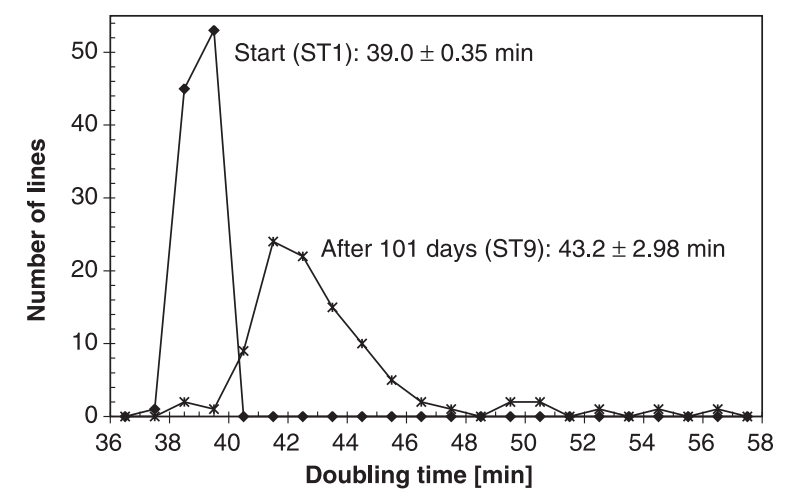

Fig. 1. The distribution of doubling times in fresh medium before serial transfer 1 (ST1, serial transfer 1) and after (ST9) prolonged periods in the stationary phase. Each distribution (mean \pm standard deviation) contains one measurement of each line that experienced the corresponding serial transfer. The dilution of the inoculum was 2401-fold in all measurements. The long tail in ST9 shifts the arithmetic mean toward higher doubling times. 
magnitude less than the deleterious mutation rate found here. Assuming either 25 generations per day in stationary phase or that all mutations are slightly deleterious $\left[\mathrm{U}_{\text {total }}\right.$ of 0.0025 (3)] yields a rate that is still less than one-fifth of what we observe. To explain our values, we would have to assume constant log-phase growth and a mutator phenotype $(11,28)$, which is not a very plausible scenario for a nonmutator strain in stationary phase. The mutation rates of three randomly chosen lines did not show differences compared with the founder strain (SOM Text).

During stationary phase, at least in one assay system, a subpopulation of cells can turn into transient mutators $(17,29)$, producing mutations that confer a growth-promoting or adaptive phenotype. Fixation of an adaptive mutation fixes all other (potentially deleterious) mutations linked to it. This hitchhiking effect suggests a large impact of transient mutators on apparent population-wide substitution rates.

Bull et al. (30), using the Torkelson et al. data from $E$. coli (17), estimated a mutation rate that is equivalent to 0.00035 mutations per day per mutational target after a 4-day starvation course at $37^{\circ} \mathrm{C}$. If one assumes that the genome of $E$. coli contains about 85 $(\cong 0.03 / 0.00035)$ mutational targets with similar properties that affect growth rate, then an overall mutation rate of $\sim 0.03$ per genome per day results. This mutation rate was observed in a small fraction of a starving stationary phase culture of normal cells of $E$. coli but not in long-term mutators. The general phenomenon of transient mutability induced by starvation has recently been shown to occur in most (but not all) natural isolates of E. coli (12). Cells that survive the longest in such experiments may do so because their transiently high mutation rates repeatedly generate selected phenotypes. If all other cells die, the effective population size in the stationary phase might be much smaller than one would assume initially. Resulting hitchhiking events may significantly accelerate evolution in the stationary phase. Thus, it appears that mutagenesis in response to starvation might generate many more mutations than DNA replication errors that occur during cell division.

This suggests a correlation between deleterious mutation rate estimates and the time bacteria spend in the stationary phase in mutation accumulation experiments. Support comes from a comparison of our study with the two replication-based dele-
Fig. 2. BM analysis of the stationary phase mutation accumulation experiment. This plot corresponds to a deleterious mutation rate $U_{B M}$ of 0.026 per genome per day with a deleterious selection coefficient $s_{B M}$ of $3.5 \%$, assuming mutational effects are equal. All growth rates $(m)$ of this plot were observed at a 2401-fold dilution. The solid line and circles denote means (M); the dashed line and diamonds denote variances (V). The highest and lowest growth

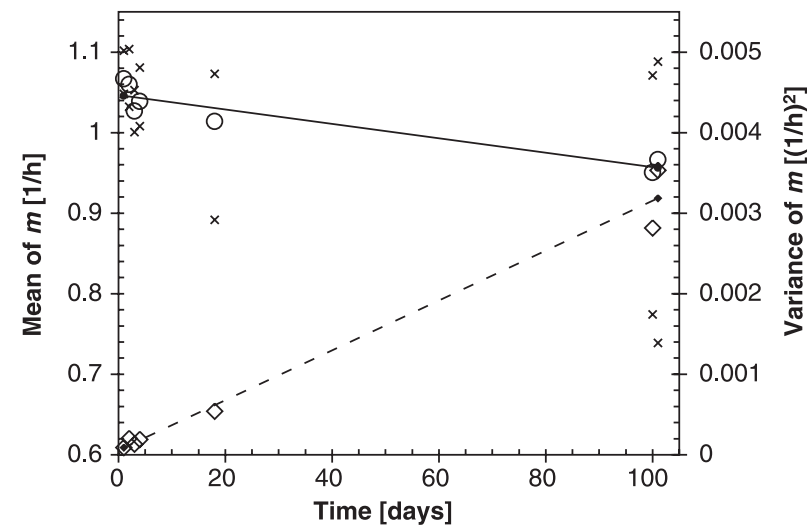

rates are marked $(\times)$. From the slope of the regression lines, $U_{B M}=(\Delta M)^{2} / \Delta V$ and $s_{B M}=\Delta V / \Delta M$ were calculated, where $\Delta$ is the difference for 1 day (9).

Fig. 3. Mutation rates per generation depend on time spent in stationary phase. All estimates have been derived from descendants of the same strain of Escherichia coli growing at $37^{\circ} \mathrm{C}$. The lowest mutation rate was observed over 7500 generations in 300 days (9), the highest rate in Fig. 2. The link between these extremes comes from 10,000 generations of mutation accumulation in genes not required for metabolism in Lenski's longterm serial transfer evolution experiment [data for BatemanMukai analysis could be estimated from figure 4 in (10)]. $U$ denotes BM estimates of deleterious genomic mutation rates. Error bars, estimated range of values. terious mutation accumulation experiments available that used the same strain, i.e., REL606 (9, 10). It is striking (Fig. 3) that a $\mathrm{BM}$ analysis of the data reported by Cooper and Lenski (6.7 generations per day at $37^{\circ} \mathrm{C}$ in liquid glucose minimal medium) led to a higher deleterious mutation rate than the one found by Kibota and Lynch (25 generations per day at $37^{\circ} \mathrm{C}$ on modified Davis minimal agar plates).

Mutation rates in stationary phase are likely to be influenced by (i) temperaturedependent biochemical reactions, (ii) the genetic background of the strain (12), and (iii) the complex population genetics of stationary phase that determines the fixation probability of mutants in the population. Our data indicate that the time bacteria spend in stationary phase must be very important for their evolution and, given the ubiquity of stationary phase or near-stationary phase conditions in nature, our results suggest we should reshape our views about bacterial evolution (31). Although such high mutation rates can be deadly for bacteria caught in stationary phase, they probably play an important role in escaping local extinction, accelerating adaptation (32), and evolving pathogenicity (33).

References and Notes

1. T. Mukai, Genetics 50, 1 (1964).

2. M. Lynch et al., Evolution 53, 645 (1999).

3. J. W. Drake, B. Charlesworth, D. Charlesworth, J. F. Crow, Genetics 148, 1667 (1998).

4. P. D. Keightley, A. Eyre-Walker, Genetics 153, 515 (1999).

5. E. Domingo, Virology 270, 251 (2000).

6. C. Zeyl, J. DeVisser, Genetics 157, 53 (2001).

7. K. Szafraniec, R. H. Borts, R. Korona, Proc. Natl. Acad. Sci. U.S.A. 98, 1107 (2001).

8. D. I. Andersson, D. Hughes, Proc. Natl. Acad. Sci. U.S.A. 93, 906 (1996).

9. T. T. Kibota, M. Lynch, Nature 381, 694 (1996).

10. V. S. Cooper, R. E. Lenski, Nature 407, 736 (2000).

11. P. Funchain et al., Genetics 154, 959 (2000).

12. I. Bjedov et al., Science 300, 1404 (2003).

13. R. Y. Morita, Bacteria in Oligotrophic Environments: Starvation-Survival Lifestyle (Chapman \& Hall, New York, 1997).

14. S. E. Finkel, E. R. Zinser, R. Kolter, in Bacterial Stress Responses, G. Storz, R. Hengge-Aronis, Eds. (ASM Press, Washington, DC, 2000), pp. 231-238.

15. H. J. Bull, G. J. McKenzie, P. J. Hastings, S. M. Rosenberg, Genetics 154, 1427 (2000).

16. W. A. Rosche, P. L. Foster, Proc. Natl. Acad. Sci. U.S.A. 96, 6862 (1999).

17. J. Torkelson et al., EMBO J. 16, 3303 (1997).

18. S. E. Finkel, R. Kolter, Proc. Natl. Acad. Sci. U.S.A. 96, 4023 (1999).

19. H. J. Bull, M. J. Lombardo, S. M. Rosenberg, Proc. Natl. Acad. Sci. U.S.A. 98, 8334 (2001).

20. B. Yeiser, E. D. Pepper, M. F. Goodman, S. E. Finkel, Proc. Natl. Acad. Sci. U.S.A. 99, 8737 (2002).

21. A. Matin, E. A. Auger, P. H. Blum, J. E. Schultz, Annu. Rev. Microbiol. 43, 293 (1989).

22. H. Ochman, S. Elwyn, N. A. Moran, Proc. Natl. Acad. Sci. U.S.A. 96, 12638 (1999).

23. F. J. Ryan, T. Okada, T. Nagata, J. Gen. Microbiol. 30, 193 (1963).

24. F. J. Ryan, D. Nakada, M. J. Schneider, Z. Vererbungsl. 92, 38 (1961).

25. Materials and methods are available as supporting material on Science Online.

26. P. D. Keightley, Genetics 150, 1283 (1998). 
27. S. F. Elena, R. E. Lenski, Nature 390, 395 (1997).

28. P. D. Sniegowski, P. J. Gerrish, R. E. Lenksi, Nature 387, 703 (1997)

29. S. M. Rosenberg, C. Thulin, R. S. Harris, Genetics 148 , 1559 (1998).

30. H. J. Bull, G. J. McKenzie, P. J. Hastings, S. M. Rosenberg, Genetics 156, 925 (2000).

31. M. Kivisaar, Environ. Microbiol. 5, 814 (2003).

32. J. P. Townsend, K. M. Nielsen, D. S. Fisher, D. L. Hartl, Genetics 164, 13 (2003).
33. J. Hacker, U. Hentschel, U. Dobrindt, Science 301, 790 (2003).

34. We thank E. Lang-Halter for excellent technical assistance, J. Blanchard and M. Lynch for providing the strain used in this study (and the NIH for the grant to M.L. that allowed J.B. to obtain the strain), P. Keightley for providing the code for ML analysis (with explanations on how to use it), and B. Charlesworth and two anonymous referees for significantly improving this manuscript.
Supporting Online Material

www.sciencemag.org/cgi/content/full/302/5650/1558/ DC1

Materials and Methods

SOM Text

Figs. S1 and S2

Tables S1 to S4

References

11 June 2003; accepted 14 October 2003

\section{Phosphatidylserine Receptor Is Required for Clearance of Apoptotic Cells}

\author{
Ming O. Li, ${ }^{1,3 *}$ Matthew R. Sarkisian, ${ }^{2 *}$ Wajahat Z. Mehal, ${ }^{1,4}$ \\ Pasko Rakic, ${ }^{2}$ Richard A. Flavell ${ }^{1,3} \uparrow$
}

Cells undergoing apoptosis during development are removed by phagocytes, but the underlying mechanisms of this process are not fully understood. Phagocytes lacking the phosphatidylserine receptor (PSR) were defective in removing apoptotic cells. Consequently, in PSR-deficient mice, dead cells accumulated in the lung and brain, causing abnormal development and leading to neonatal lethality. A fraction of PSR knockout mice manifested a hyperplasic brain phenotype resembling that of mice deficient in the cell death-associated genes encoding Apaf- 1 , caspase-3, and caspase- 9 , which suggests that phagocytes may also be involved in promoting apoptosis. These data demonstrate a critical role for PSR in early stages of mammalian organogenesis and suggest that this receptor may be involved in respiratory distress syndromes and congenital brain malformations.

Exposure of phosphatidylserine (PS) on the outer leaflet of the plasma membrane of apoptotic cells is considered a primary signal recognizable by phagocytes $(1-3)$. Several receptors are implicated in the recognition of PS, including lectin-like oxidized low-densi- ty lipoprotein receptor-1 (LOX-1), $\beta_{2}$-glycoprotein I $\left(\beta_{2} \mathrm{GPI}\right)$ receptor, $\alpha_{\mathrm{v}} \beta_{3}$ vitronectin receptor, Mer receptor tyrosine kinase, and PSR (4-9). In vitro, PSR is essential for the engulfment of apoptotic cells by both professional and amateur phagocytes, including macrophages, fibroblasts, epithelial cells, and endothelial cells (9, 10). We examined PSR expression in mouse embryos. Northern blot analysis indicated that PSR is expressed as early as embryonic day 7 (E7) (fig. S1A). PSR transcripts were present in multiple tissues including brain, eye, spinal cord, thymus, lung, liver, kidney, and intestine (fig. S1B).

To delete PSR in the mouse, we replaced the first two exons of the PSR gene with the neomycin resistance gene in mouse embry-

${ }^{1}$ Section of Immunobiology, ${ }^{2}$ Department of Neurobiology, ${ }^{3}$ Howard Hughes Medical Institute, ${ }^{4}$ Section of Digestive Diseases, Yale University School of Medicine, New Haven, CT 06520, USA.

*These authors contributed equally to this work. $\dagger$ To whom correspondence should be addressed. Email: richard.flavell@yale.edu
Fig. 1. Abnormal lung development and failed clearance of apoptotic cells in PSR-deficient mice. (A) Phenotype of $\mathrm{PSR}^{+/+}$and $\mathrm{PSR}^{-1-}$ mice at PO. $\mathrm{PSR}^{-1-}$ mice showed cyanotic skin color. (B) Lung from a PO $\mathrm{PSR}^{-1-}$ mouse sinks in phosphate-buffered saline; lung from a $\mathrm{PSR}^{+/+}$mouse floats. (C) Reduced lumenal air spaces in $\mathrm{PSR}^{-/-}$lung at E17.5 (upper panels, arrows) and PO (lower panels, A). At E17.5, expanded interstitial areas (I) are between type II epithelial cells (T2); at PO, lung surfactant (SF) is indicated in $\mathrm{PSR}^{+/+}$ and $\mathrm{PSR}^{-/-}$lungs. (D) Increased TUNEL-positive cells (arrows) in E17.5 $\mathrm{PSR}^{-/-}$lungs. Counterstaining with propidium iodide (PI) is also shown (lower panels). (E) EM analysis of an engulfed apoptotic cell (arrow) in $\mathrm{PSR}^{+/+}$lung (left). A nonphagocytosed, necrotic-like cell with a semicondensed nucleus and dilated nuclear membranes (arrows) and mitochondria (M) in PSR ${ }^{-1-}$ lung (right). Scale bars, $25 \mu \mathrm{m}$ (C), $2.5 \mu \mathrm{m}$ (E).

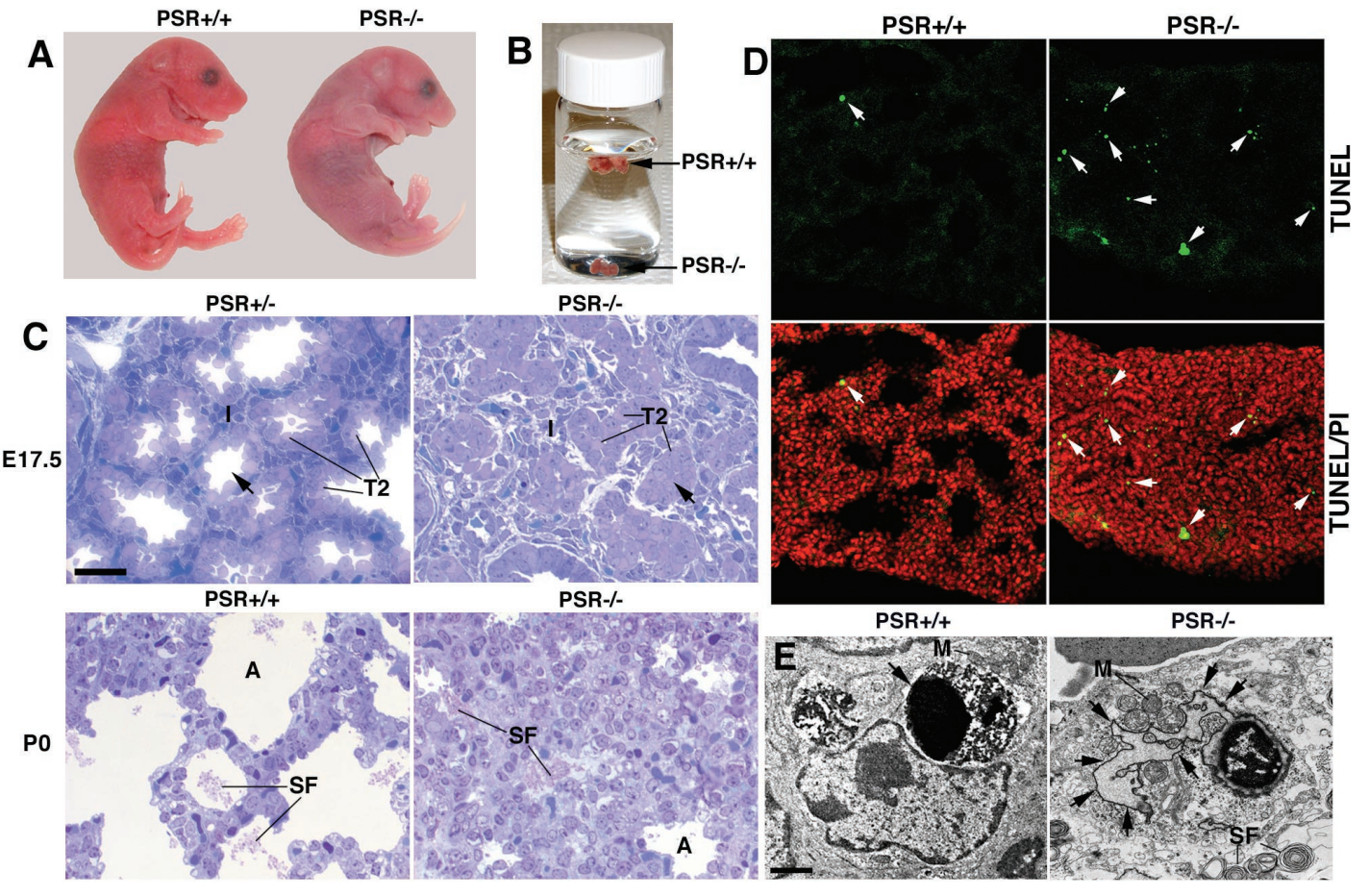

